\title{
A Method to Improve Activation of Implanted Dopants in SiC
}

O. W. Holland and D. K. Thomas

Oak Ridge National Laboratory, Oak Ridge, TN 37831-6048

\begin{abstract}
Implantation of dopant ions in $\mathrm{SiC}$ has evolved according to the assumption that the best electrical results (i.e., carrier concentrations and mobility) is achieved by using the highest possible processing temperature. This includes implantation at $>600^{\circ} \mathrm{C}$ followed by furnace annealing at temperatures as high as $1750^{\circ} \mathrm{C}$. Despite such aggressive and extreme processing, implantation suffers because of poor dopant activation, typically ranging between $<2 \%-50 \%$ with p-type dopants represented in the lower portion of this range and n-types in the upper. Additionally, high-temperature processing can led to several problems including changes in the stoichiometry and topography of the surface, as well as degradation of the electrical properties of devices. A novel approach for increasing activation of implanted dopants in $\mathrm{SiC}$ and lowering the activation temperature will be discussed. This approach utilizes the manipulation of the ion-induced damage to enhance activation of implanted dopants. It will be shown that nearly amorphous layers containing a small amount of residual crystallinity can be recrystallized at temperatures below $900^{\circ} \mathrm{C}$ with little residual damage. It will be shown that recrystallization traps a high fraction of the implanted dopant residing within the amorphous phase (prior to annealing) onto substitutional sites within the $\mathrm{SiC}$ lattice.
\end{abstract}

\section{INTRODUCTION}

Silicon carbide is a wide band-gap semiconductor that offers advantages over silicon for use in fabricating devices for high-power, high-temperature applications [1]. The ability to fabricate discrete devices and integrated circuits depends, in part, upon techniques for selective-area doping. Since the diffusivity of dopants in $\mathrm{SiC}$ is extremely low, selective-area doping by diffusion from a gaseous source is not viable. Therefore, dopant-ion implantation has come to the forefront as the technique of necessity, if not choice. However, successful implementation of implantation in $\mathrm{SiC}$ has been slow and uncertain, thus hindering efforts to develop $\mathrm{SiC}$ technology as an alternative to $\mathrm{Si}$. By comparison, implantation of Si led the revolution in manufacturing that yielded electronic circuits at unprecedented levels of integration. The problem in $\mathrm{SiC}$ is that implanted dopants are difficult to activate [2]. This has lead to a "conventional" wisdom that teaches the use of extreme measures to activate implanted dopants including the use of annealing temperatures $\geq 1600^{\circ} \mathrm{C}$, and heating the samples during implantation at $\geq 600^{\circ} \mathrm{C}$ [3]. Adherence to this wisdom does, however, create many problems. First, it is expensive and requires the development of implantation masks that can withstand irradiation at high temperature. Secondly, sublimation during high-temperature annealing disrupts the stoichiometry of the surface layer, which can lead to substantial surface roughening [4]. 
A number of reports are at odds with this processing convention. It has been demonstrated that $p$ - $n$ diodes formed by multiple-energy $\mathrm{N}^{+}$-implantation at room temperature (RT) into $p$-type epilayers function similarly to those formed by implantation at $700^{\circ} \mathrm{C}$ [5]. Secondly, electrical characterization of $4 \mathrm{H}-$ and $6 \mathrm{H}-\mathrm{SiC}$ was done to compare the effectiveness of $\mathrm{Al}^{+}$and $\mathrm{C}^{+}$coimplantation at room temperature $(\mathrm{RT})$ and $600^{\circ} \mathrm{C}$ [6]. A higher activation of Al was achieved in samples implanted at RT. This surprisingly was attributed to the greater amount of ion-induced damage formed at RT, including amorphization of the lattice. Both results are inconsistent with the need for extreme processing measures, especially the use of implantation at elevated temperature.

This paper further challenges the "conventional" wisdom relating to activation of implanted dopants in $\mathrm{SiC}$. The evolution of ion-induced damage and its morphology during irradiation will also be discussed. The results will show that the evolution of a continuous amorphous $(\alpha)$ layer occurs by overlap of isolated $\alpha$-zones. An understanding of damage and its annealing behavior make it possible to achieve dopant activation in $\mathrm{SiC}$ using less extreme implant conditions. Especially, it will be demonstrated that damage can be effectively annealed and dopants activated even for implantation at RT. This depends critically upon the damage morphology formed during irradiation. Coimplantation with an isoelectric ion such as $\mathrm{Si}^{+}$to manipulate the damage morphology will be discussed. These results will challenge the "conventional" wisdom, and ultimately require it to be discarded or substantially qualified.

\section{EXPERIMENTAL PROCEDURE}

Implantation was done with a raster-scanned beam at an average current density of $\sim 0.2 \mu \mathrm{amps} / \mathrm{cm}^{2}$. Samples were clamped to a holder that could be resistively heated to achieve the desired implantation temperature. 4H-SiC (1000) wafers used in the study were n-type, research grade material obtained from Cree Research with a resistivity of $0.1-0.5 \Omega-\mathrm{cm}$.

Samples were analyzed using Rutherford backscattering/channeling spectrometry (RBS), employing 2.3 $\mathrm{MeV} \mathrm{He}^{++}$-ions and a solid-state, surface-barrier detector positioned at $160^{\circ}$. Channeling spectra were acquired with the sample aligned along the [1000] axis normal to the surface. Spectra were normalized using standard current integration techniques. Two different spectral components, direct scattering and dechanneling, were utilized to monitor ion-induced damage and its annealing behavior. The interaction of channeled ions with displaced atoms results in either (1) direct scattering as a result of large-angle collisions, or (2) dechanneling. Dechanneling occurs when an ion suffers a small-angle collision and is scattered outside the critical angle for channeling. Since the direct scattering and dechanneling occur at defects within the lattice, they both can be used as a quantitative measure of the total damage.

Deconvolution of the aligned yield into direct scattering and dechanneling is illustrated in Fig. 1. While both yields correlate closely with the damage concentration, they are affected differently by its morphology. In general, more direct scattering occurs from displaced atoms randomly distributed in isolated $\alpha$-zones than located at correlated sites within a dislocation. The converse is true for dechanneling [7]. Therefore, monitoring both yields provides information on the amount and type of damage present in a sample. 
Samples were annealed in a standard tube furnace under flowing Ar for 15 min. after reaching temperature, as monitored by a thermocouple in the furnace. Anneals were done at either $900^{\circ}$ or $1200^{\circ} \mathrm{C}$.

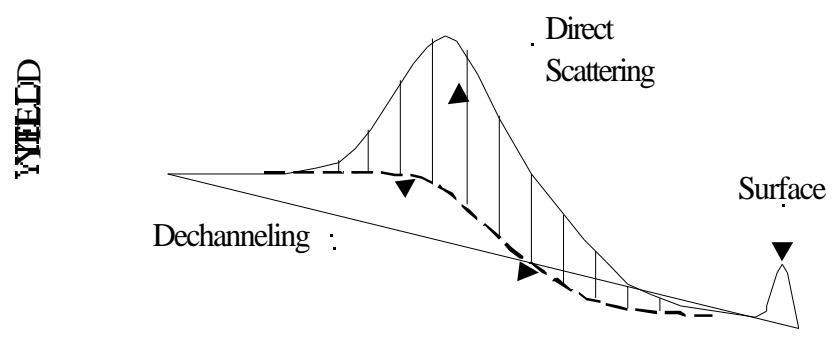

BACKSCATTERED ENERGY

Figure 1. Illustration of a backscattered-energy spectrum of channeled ions from an implanted sample. The scattering peak below the surface reflects the presence of damage at that location. The deconvolution of the aligned yield in this region into its components, direct scattering and dechanneling, is shown.

\section{RESULTS AND DISCUSSION}

The Morphology and Annealing Behavior of Ion-Induced Damage:

It is instructive to investigate the annealing behavior of damage formed during implantation of SiC. Aligned spectra in Fig. 2 are shown from samples implanted with $190 \mathrm{keV} \mathrm{Si}^{+}$-ions at RT for different doses. Spectra were acquired both after implantation, as well as annealing at $900^{\circ}$ and $1200^{\circ} \mathrm{C}$. It is immediately clear that the annealing behavior in the samples is quite different. At a dose of $8 \times 10^{15} \mathrm{~cm}^{-2}$, the aligned yield from the as-implanted sample is at the random level over the range of the ions, $R_{p}$. This is consistent with the formation of a continuous $\alpha$-layer extending from the surface to well within the sample at $\sim 1.12 \mathrm{MeV}$. The only variation in the aligned yield after annealing occurs in the region near the original interface, otherwise the yield remains at the random level. This indicates that the lattice has been restored locally in the interfacial region to nearly defect-free, single-crystal. However, the remainder of the implanted region is not in registry with the bulk. Annealing appears to have resulted in formation of randomly oriented polycrystals, consistent with the results of Höfgen et al. [8] that showed crystallization of $\alpha$-layer in SiC yields a polycrystal with different polytypes. Even after annealing at $1200^{\circ} \mathrm{C}$, there appears to be little preferential alignment of the polycrystals with the bulk except a small amount of epitaxial growth in the interfacial region. At a lower fluence of $5 \times 10^{14} \mathrm{~cm}^{-2}$, the situation is quite different. The large damage peak in the as-implanted spectrum is clear evidence of significant disorder in the lattice but clearly below the random level (which would indicate complete amorphization). In addition, annealing has a dramatic effect upon this damage. A large decrease in the scattering yield from the implanted region after $900^{\circ} \mathrm{C}$ annealing is clear evidence that much of the damage is removed at this temperature. Further annealing at $1200^{\circ} \mathrm{C}$ decreases the yield even more. Therefore, ion-induced damage below the level 
of complete amorphization is readily removed in $\mathrm{SiC}$ by annealing at relatively modest temperatures.

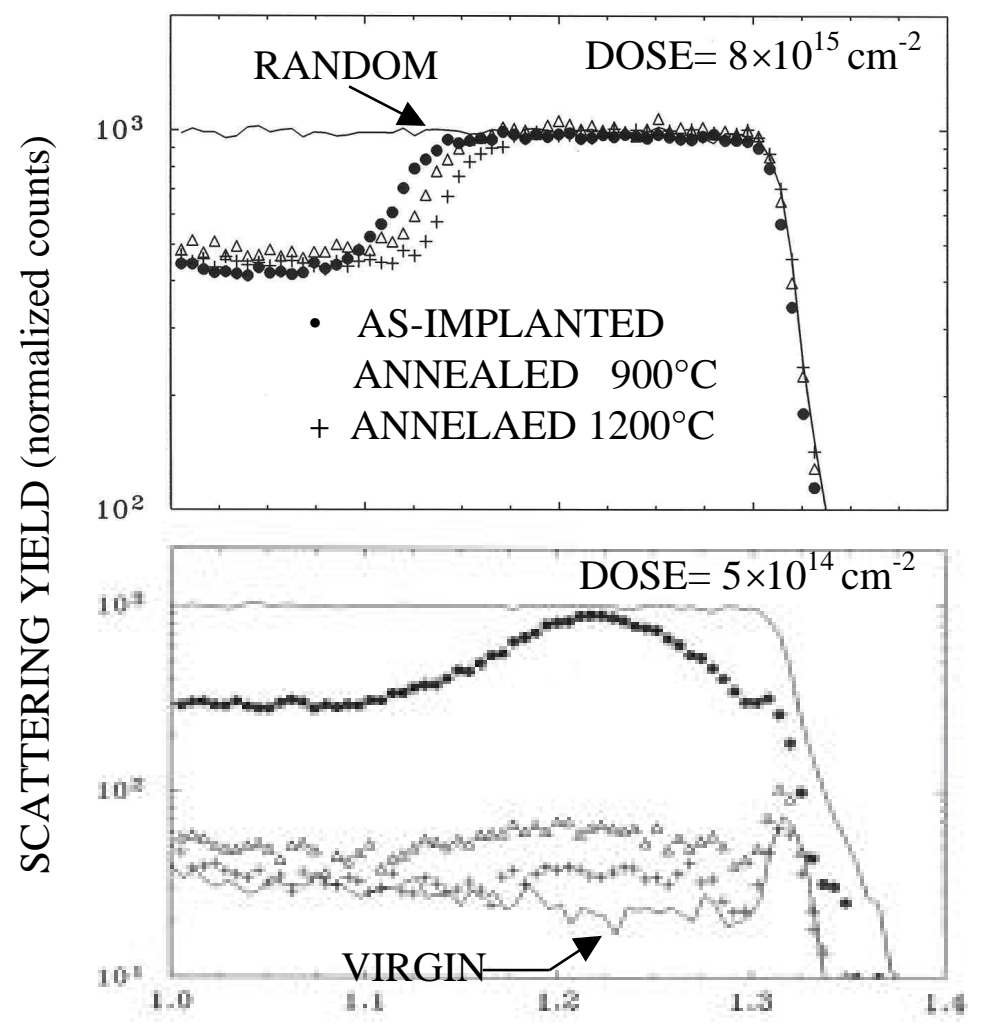

BACKSCATTERED ENERGY (MeV)

Figure 2. Channeling spectra from 4H-SiC implanted at RT with different doses of 190 $\mathrm{keV} \mathrm{Si}{ }^{+}$-ions demonstrating the effects of annealing at $900^{\circ}$ and $1200^{\circ} \mathrm{C}$. A random and an aligned virgin spectrum are shown for reference.

It is important to determine whether or not high temperature implantation offers any advantage over RT in yielding a more defect-free process. While damage will undoubtedly be lower in samples implanted at high temperature, this is not the critical issue but rather the final state of the lattice after furnace annealing. The annealing behavior of damage formed during $190 \mathrm{keV} \mathrm{Si}^{+}$-implantation at different temperatures is shown in Fig. 3. Comparison of the as-implanted damage levels for the three different temperatures indicates that substantial dynamic annealing occurs during a $250^{\circ} \mathrm{C}$ irradiation. However, the damage levels converge after furnace annealing such that there is little or no difference in the amount of residual damage after $1200^{\circ} \mathrm{C}$ (e.g. compare the damage level after $1200^{\circ} \mathrm{C}$ annealing in samples implanted at RT and $600^{\circ} \mathrm{C}$ ). Therefore, high temperature implantation seems to offer little advantage over RT. Even though the damage levels may initially be greater (after RT implantation), the final state after furnace annealing appears to be independent of the implantation conditions. However, this statement is true only for conditions that do not yield a continuous $\alpha$-layer. Given 
the difficulty in restoring the $\mathrm{SiC}$ lattice after amorphization, there is a clear benefit to using high temperature implantation to suppress this effect.

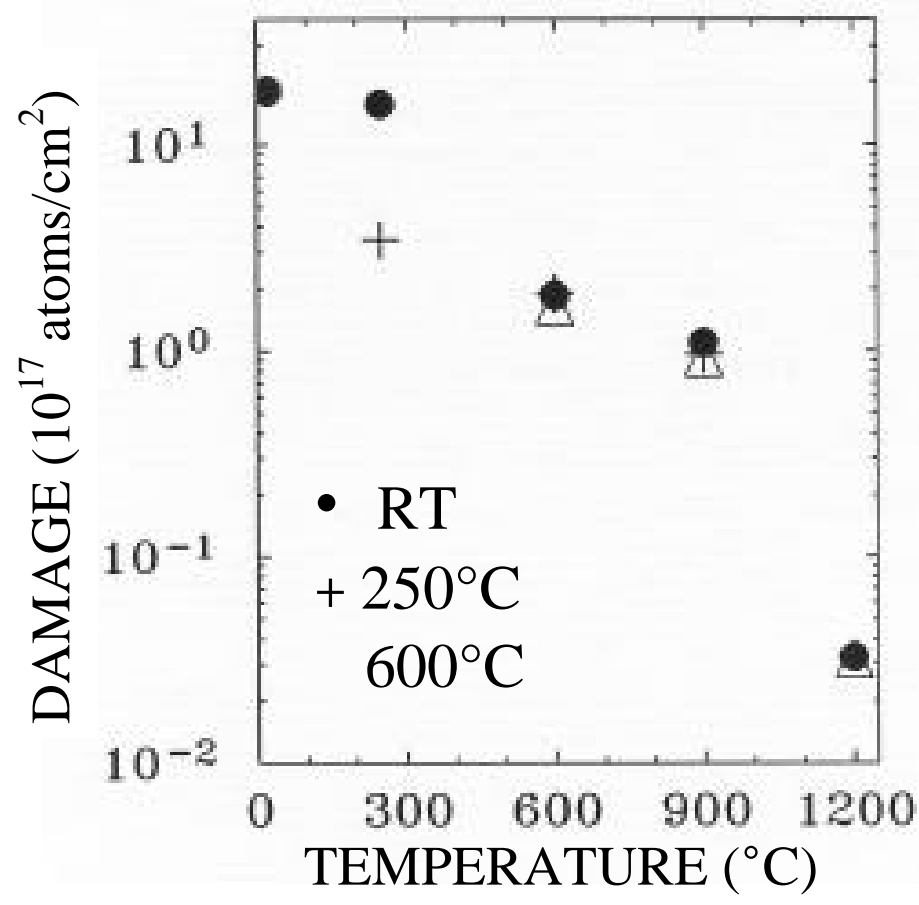

Figure 3. Residual damage as a function of annealing temperature for samples implanted with $190 \mathrm{keV} \mathrm{Si} i^{+}$-ions to a dose of $5 \times 10^{14} \mathrm{~cm}^{-2}$ at $\mathrm{RT}, 250^{\circ}$ and $600^{\circ} \mathrm{C}$, respectively.

Although damage formed at RT can be adequately annealed, it is somewhat surprising since annealing of structural defects generally involves the motion of point defects. Mechanisms such as dislocation climb or dissociation of defect clusters require the action of mobile point defects. However, $\mathrm{SiC}$ is a high-temperature material and, as such, diffusion within the lattice is negligible, especially at temperatures used in this study. Furthermore, dynamic effects in SiC during implantation appear to be unrelated to long-range motion of defects. This was demonstrated by studying the effect of dose-rate on damage formation. Dose rate is simply defined as the arrival rate of the ions (i.e. the ion current). Dose-rate effects have been widely studied in other semiconductors, such as $\mathrm{Si}$ [9], Ge [10], SiGe [10], and GaAs [11,12], where damage was generally observed to increase with dose-rate (at a fixed dose). Such effects arise when damage is formed by a nucleation and growth process involving the interaction of mobile defects within the irradiated volume. This is referred to as a homogeneous mechanism [13]. However, no dose-rate effects were observed in $\mathrm{SiC}$ for $190 \mathrm{keV} \mathrm{Si}^{+}$-implantation at RT. This strongly indicates that point defects are immobile at this temperature. Therefore, damage must form heterogeneously within the collision cascade of the ion during its quench. Surprisingly, no dose-rate effects were observed even during $250^{\circ} \mathrm{C}$ irradiation despite substantial dynamic annealing. Dynamic annealing at this temperature must not involve long-range diffusion of defects but rather local reordering of the defect structure. This is 
consistent with the formation of isolated amorphous zones. These zones can be formed within a collision cascade when the density of displaced atoms is sufficiently large to initiate a spontaneous crystal-to- $\alpha$ transition. (A continuous $\alpha$-layer will form when overlap of the isolated zones is sufficient to completely fill the volume.) Furthermore, these zones are easily annealed by solid-phase, epitaxial growth (SPEG), which only involves atomic rearrangement at the $\alpha$-interface [14]. This situation is identically to what occurs in Si irradiated with self-ions at cryogenic temperatures to suppress defect mobility [14]. In this case, no dose-rate effects were observed and electron microscopy confirmed the defect morphology to consist of $\alpha$-zones.

Analysis of the aligned spectra from samples implanted at RT confirms this speculation. In particular, the ratio of direct scattering to dechanneling was determined over a wide range of dose spanning damage levels near virgin all the way to complete amorphization of the lattice. The results are shown in Fig. 4 and indicate a nearly constant ratio over the entire dose range for the $190 \mathrm{keV} \mathrm{Si}{ }^{+}$-ion irradiation. Since dechanneling and direct scattering are affected differently by the damage morphology, the constant ratio strongly indicates that the morphology is not changing over the dose range. Since a continuous $\alpha$-layer was formed at the highest dose studied, it is reasonable to conclude that ion-induced damage formed at the lower doses consisted primarily of isolated $\alpha$-zones.

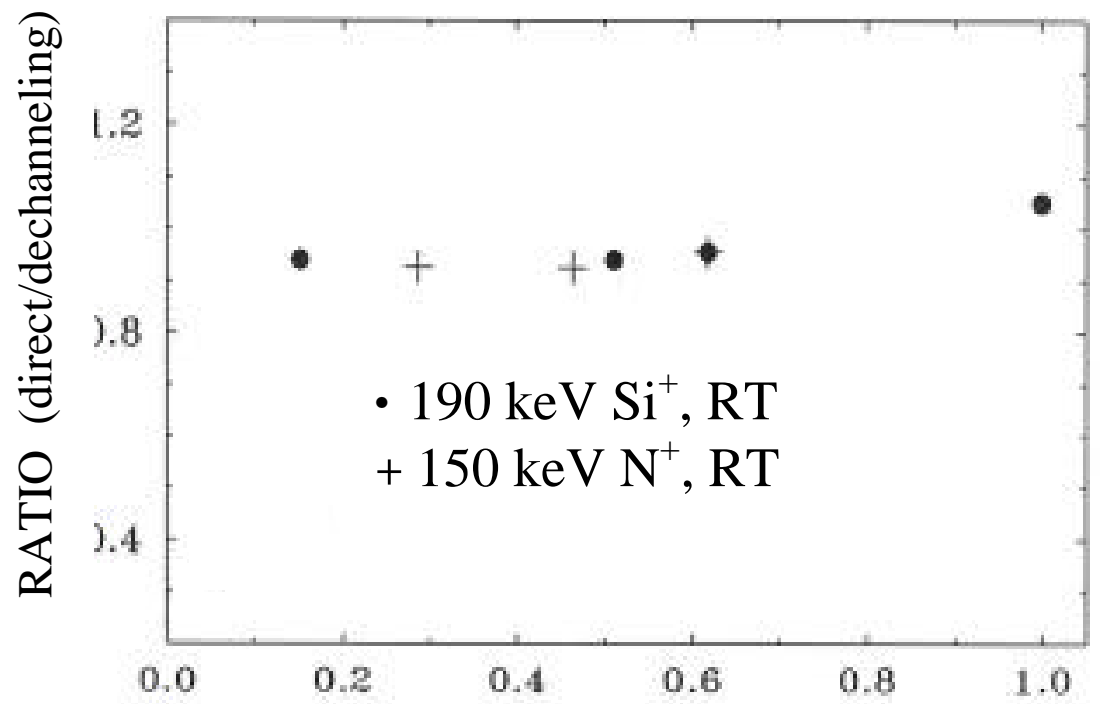

NORMALIZED DAMAGE

Figure 4. The ratio of the direct scattering yield to dechanneling from aligned spectra acquired from $4 \mathrm{H}$-SiC samples implanted under various conditions. The ratio is seen to be fairly constant for both $\mathrm{N}^{+}$- and $\mathrm{Si}^{+}$-ion over a wide range of as-implanted damage (i.e. dose), thus indicating the damage morphology is similar under these conditions. 


\section{A Technique for Improved Dopant Activation:}

Conventional processing suffers due to poor dopant activation. However, the previous results suggest that, instead of using extreme measures to avoid damage accumulation, it may be possible to utilize damage to assist in dopant activation. Although this approach is a radical departure from standard $\mathrm{SiC}$ processing, it is based in fundamental principles utilized in Si processing. Amorphization, either directly by dopant implantation or by using an isoelectric species prior to dopant irradiation, has been widely utilized in $\mathrm{Si}$ to improve dopant activation [15]. Amorphous layers in Si crystallize during annealing by SPEG at the crystal- $\alpha$ interface to yield defect-free, single-crystal with the implanted dopant trapped substitutionally within the lattice, even at concentrations far exceeding solid solubility. Unfortunately, bulk nucleation during recrystallization of $\alpha$-layers in $\mathrm{SiC}$ leads to the formation of a polycrystalline phase consisting of misoriented grains of different $\mathrm{SiC}$ polytypes [8]. In addition, stress generated during recrystallization leads to severe cracking within the layer extending into the substrate (substantially beyond the depth of the amorphous interface) and partial exfoliation of the surface.

However, it is possible to recover the benefits of amorphization in $\mathrm{SiC}$ using a slight variation of the method. As discussed earlier, amorphous layers can be formed in $\mathrm{SiC}$ that contain a small amount of residual crystallinity distributed throughout the layer. Such layers crystallize at $900^{\circ} \mathrm{C}$ with complete alignment to the substrate and with little residual damage. Presumably, this crystallization occurs by SPEG at the interface between the imbedded amorphous regions and the crystalline matrix. As such, it should trap the portion of the implanted dopant residing within the imbedded amorphous phase (prior to annealing) onto substitutional sites within the $\mathrm{SiC}$ lattice.

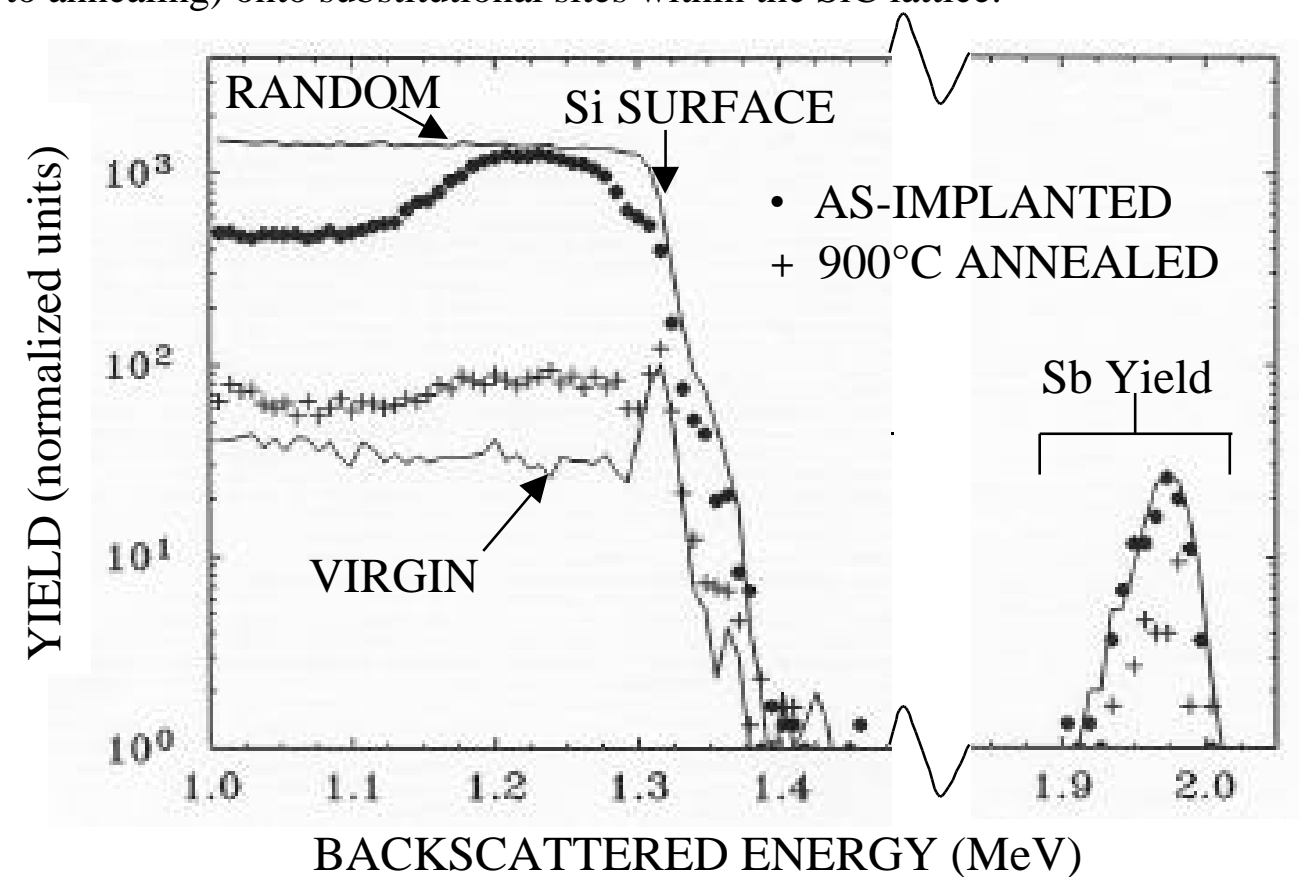

Figure 5. Spectra from $\mathrm{SiC}$ coimplanted with $\mathrm{Sb}^{+}-\left(200 \mathrm{keV}, 5 \times 10^{14} \mathrm{~cm}^{-2}, 600^{\circ} \mathrm{C}\right)$ and $\mathrm{Si}^{+}$-ions $\left(190 \mathrm{keV}, 5 \times 10^{14} \mathrm{~cm}^{-2}, R T\right)$. Also, an aligned spectrum from a virgin sample and a random from the coimplanted sample are shown for reference. 
To demonstrate this technique, $\mathrm{Sb}$ was implanted at high-temperature to maintain the crystallinity of the irradiated volume. Subsequently, the sample was implanted with 190 $\mathrm{keV} \mathrm{Si}^{+}$-ions at RT to a dose of $5 \times 10^{14} \mathrm{~cm}^{-2}$ to create a high volume fraction of amorphous material within the Sb-doped region. The spectral results are shown in Fig. 5. The scattering from $\mathrm{Si}$ at the surface $(\sim 1.32 \mathrm{MeV})$, and the $\mathrm{Sb}^{+}$-implanted region (1.9$2.0 \mathrm{MeV}$ ) are marked in the figure for easy identification. First, notice that the aligned yield from $\mathrm{Sb}$ in the as-implanted sample is similar to that in the random spectrum indicating a high degree of amorphization. Thus, coimplantation has left the $\mathrm{Sb}$ randomly distributed within a partially amorphized lattice. Annealing at $900^{\circ} \mathrm{C}$ results in significantly less scattering from both $\mathrm{Si}$ and $\mathrm{Sb}$ atoms in the implanted region, indicating that the lattice has been largely restored, and much of the $\mathrm{Sb}$ has been incorporated onto substitutional sites. The substitutional fraction is shown in Fig. 6 to be $\geq 70 \%$ over the entire $\mathrm{Sb}$ distribution. Thus, this demonstrates that partial amorphization of the $\mathrm{SiC}$ lattice is a viable technique for activating implanted dopants. Clearly, more work needs to be done, in particular electrical measurements are needed to verify the ion scattering results.

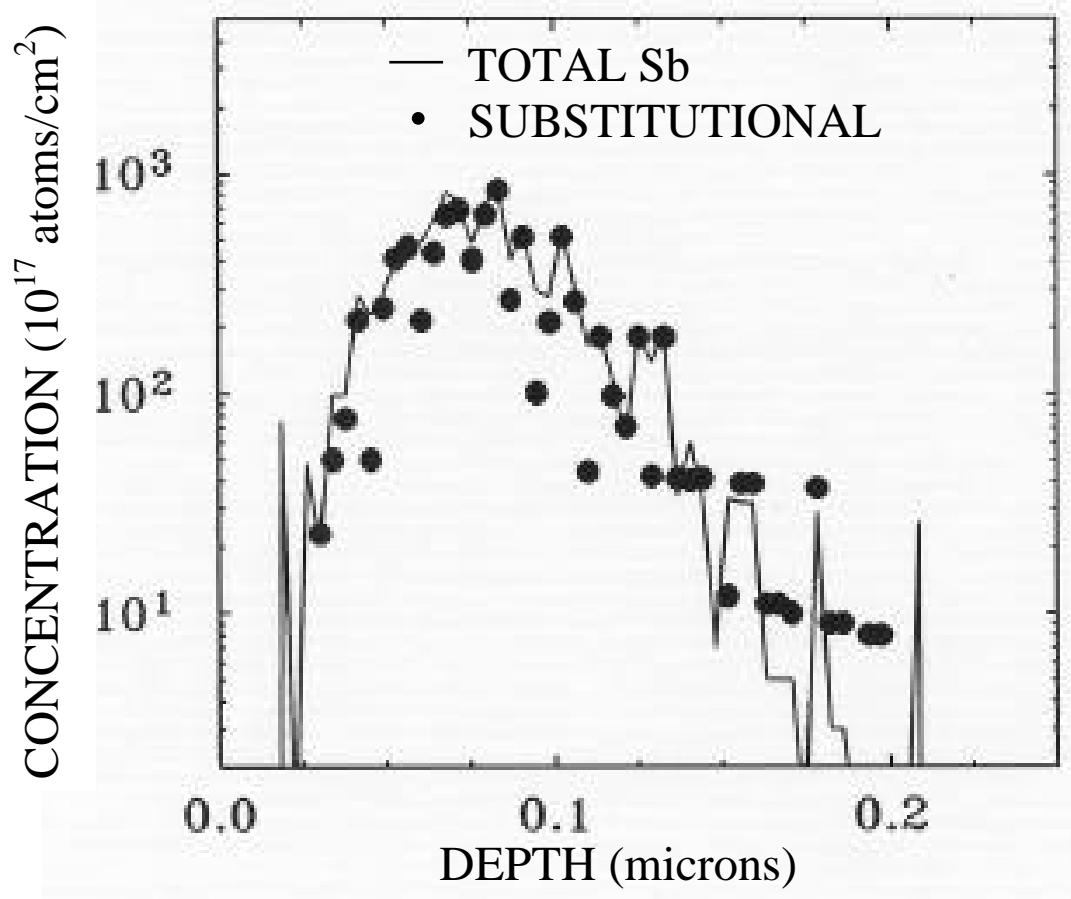

Figure 6. Total and substitutional concentrations of implanted $\mathrm{Sb}$ after $900^{\circ} \mathrm{C}$ annealing.

\section{CONCLUSIONS}

Ion-induced damage was shown to consist predominantly of isolated $\alpha$-zones in $\mathrm{SiC}$ irradiated with moderately heavy ions at RT. These zones anneal at $\leq 900^{\circ} \mathrm{C}$, presumably by a SPEG mechanism that yields almost complete recovery of the lattice. Furthermore, 
it was demonstrated that dopant atoms within the $\alpha$-zones are trapped onto substitutional sites during SPEG. Thus, it may be possible to use this technique to achieve better activation of light dopant atoms, such as boron. Little or no activation of implanted boron occurs during annealing at $\leq 1600^{\circ} \mathrm{C}$, irrespective of the implantation temperature [16]. Coimplantation with $\mathrm{Si}^{+}$-ions to partially amorphize the $\mathrm{B}^{+}$-implanted region should activate a large fraction of the boron at $\leq 900^{\circ} \mathrm{C}$.

\section{ACKNOWLEDGEMENTS}

Research Sponsored by the Division of Material Sciences, U.S. Department of Energy, under contract DE-AC05-00OR22725 with Oak Ridge National Laboratory, managed by UT-Battelle, LLC.

\section{REFERENCES}

1 "Materials for High Temperature Semiconductor Devices," by the Committee on Materials for High Temperature Semiconductor Devices, NMAB-747, (National Academy Press, Washington, 1995).

2 See for example "Fundamentals of SiC-Based Device Processing," M. R. Melloch and J. A. Cooper, Jr., MRS Bulletin, March 1997, p. 42, and references within.

3. "Implantation and Electrical Activation of Dopants into Monocrystalline Silicon Carbide," John A. Edmond and Robert F. Davis, U.S. Patent 5,087,576, 1992.

4. M. A. Capano, S. Ryu, M. R. Melloch, J. A. Cooper, Jr., and M. R. Buss, J. Electron. Mater. 27, 370 (1998); and Mulpuri V. Rao, Jesse B. Tucker, M. C. Ridgway, O. W. Holland, N. Papanicolau and J. Mittereder, J. Appl. Phys. 86, 752 (1999).

5. Jason A. Gardner, Andrew Edwards, Mulpuri V. Rao, N. Papanicolaou, G. Keiner, O. W. Holland, M.A. Capano, M. Ghezzo, and J. Kretchmer, J. Appl. Phys. 83, 5118 (1998).

6. Kiyoshi Tone, IEEE Transactions on Electron Devices, 46, 612 (1999).

7. W. F. Tseng, J. Gyulai, T. Koji, S. S. Lau, J. Roth, and J. W. Mayer, Nucl. Instr. and Meth. 149, 615 (1978).

8. A. Höfgen, V. Heera, F. Eichhorn, and W. Sorupa, J. Appl. Phys. 84, 4769 (1998).

9. O. W. Holland, D. Fathy, J. Narayan, and O. S. Oen, Radiat. Eff. 90, 127 (1985).

10. T. E. Haynes and O. W. Holland, Nucl. Instr. and Meth. B80/81,901 (1993).

11. F. G. Moore, H. B. Dietrich, E. A. Dobisz, and O. W. Holland, Appl. Phys. Lett. 57, 911 (1990).

12. T. E. Haynes and O. W. Holland, Appl. Phys. Lett. 59, 452 (1991).

13. Lewis T. Chadderton, Radiat. Eff. 8, 77 (1971).

14. O. W. Holland, C. W. White, M. K. El-Ghor, and J. D. Budai, J. Appl. Phys. 68, 2081 (1990).

15. J. Narayan and O. W. Holland, Phys. Status Solidi A, 73 (1982) 225.

16. Mulpuri V. Rao, Jason A. Gardner, P. H. Chi, O. W. Holland, G. Kelner, J. Kretchmer and M. Ghezzo, J. Appl. Phys. 81, 6635 (1997). 